\title{
Nonlinear-optical processes at streamer discharge in semiconductors
}

\author{
K.I. Rusakov ${ }^{1}$ and V.V. Parashchuk ${ }^{2}$ \\ ${ }^{1}$ Brest State Technical University \\ ${ }^{2}$ Stepanov Physics Institute of BNSA, Minsk \\ E-mail: k.rusakov@tut.by,v.parashchuk@ifanbel.bas-net.by
}

\begin{abstract}
The possibility of light auto-channelling (self-trapping) in conditions of streamer discharge in hexagonal and cubic semiconductors was shown. It is considered the mechanism of discharge in the wide-gap compounds on the basis of representation about the light auto-channelling at streamer excitation, providing their high propagation velocity down to $\sim 5 \cdot 10^{9} \mathrm{~cm} / \mathrm{s}$, the crystallographic orientation (directionality), filamentary character at thickness of the channel about $1 \mu \mathrm{m}$ and absence of the catastrophic destructions of a crystal.
\end{abstract}

Keywords: power semiconductor streamer laser, effect of light auto-channelling (selftrapping), nonlinearity, combined effect, symmetry of crystallographic directions of system of streamer discharges.

Manuscript received 31.07.14; revised version received 12.11.14; accepted for publication 19.02.15; published online 26.02.15.

Discovery of streamer discharge in semiconductors accompanied by intensive laser effect (1973, [1]) has stimulated, on the one hand, further development of rupture and pre-rupture physics in solid body strong fields and, on the other hand, contributed to appearance of new scientific branchs - physics of semiconductors streamer discharge and streamer laser physics. At present, one of the trends in semiconductor physics and engineering is design of future-technology transistors, integrated microcircuits (optoelectronic systems) and new elemental base of opto-acousto-electronics on the basis of direct-band materials with a wide range of bandgaps. The role of emissive, including nonlinear, optical processes is great for the combinations mentioned, they influence sufficiently, therefore the question on phenomena in strong electric fields and interaction of optical and electric fields is urgent. In this relation, it seems necessary to investigate the corresponding processes at streamer discharge in widegap semiconductors, which is explained by original properties and sophistication of the phenomenon, possibility to obtain new information on substance structure and prospects for wide practical application.

Peak values of light field intensity in the channel of streamer discharge amounts $\sim 10^{9} \ldots 10^{11} \mathrm{~W} / \mathrm{cm}^{2}$ and is sufficient for different nonlinear optical effects in semiconductors, radiation self-influence in autochannelling (self-trapping) mode, in particular [2]. However, under these conditions, including media with cubic $\left(n_{2}\right)$ and of the fifth order $\left(n_{4}\right)$ nonlinearity, $n_{2}, n_{4}$ coefficients and other parameters of the problem involved - wavefront radius, size and character of initial beam (two- or three-dimensional case), are not known in details. Therefore, numerical simulation of the process is needed. Threshold, energy and special properties of the effect are analyzed below depending on nonlinearity magnitudes and other parameters for hexagonal and cubic semiconductors, the conditions of optimal autochannelling are considered.

In wide-gap compounds of $\mathrm{A}_{2} \mathrm{~B}_{6}$ type (hexagonal $\mathrm{CdS}, \mathrm{CdSe}$ and cubic CdTe) the effects of self-influence have complex nature depending on pulse durability, way 
of excitation (one-photon, two-photon) and other factors. These effects are revealed in process competition of selffocusing and defocusing [3,4]. In this case, $n_{2}+n_{4}$ $\left(n_{2}>0, n_{4}<0\right)$ nonlinearity combined effect is realized. For the mentioned media, the theory of self-channelling for intensive light beams has been developed [2], its results are used in the present work.

Our analysis of current data on light self-influence indicates the necessity of numerical simulation for the self-channelling radiation effect in discharge conditions at varying nonlinearity coefficients within the range $n_{2}$ $10^{-9} \ldots 10^{-8}$ units CGSE, $n_{4} \sim-\left(10^{-14} \ldots 10^{-12}\right)$ units CGSE. The effect criteria, including diffraction, self-focusing and self-defocusing of light, can be written in the following way:

$$
\left(2\left|n_{2} E^{2}+n_{4} E^{4}\right| / n_{0}\right)^{1 / 2}=1.22 \lambda /\left(D n_{0}\right)
$$

where $D$ is the initial beam diameter, $\lambda$ is the wavelength in vacuum, $E$ is the amplitude of electric field intensity of light wave, $n_{0}$ is linear factor of medium refraction. The relevant (1) radiation power is equal to

$P_{c r-4}=\mid c D /\left(64 n_{4}\right) \times$

$\times\left\{\left[\left(n_{0} n_{2} D\right)^{2} \pm 2 \cdot 1.22^{2} n_{0} n_{4} \lambda^{2}\right]^{1 / 2} \pm n_{0} n_{2} D\right\} \mid$.

The analysis of possible $P_{\mathrm{cr}}$ values according to (2) shows that the minimal effect threshold $P_{\mathrm{cr} 1}$ corresponds to the "-" sign in both dyads of the latter expression. Similarly, denote the power corresponding to the sign "+" in dyads by $P_{\mathrm{cr} 2}$, and $P_{\mathrm{cr} 4}$ is denoted by the signs “-”, “+”. In particular, for cadmium sulphide monocrystals in the edge region of bandgap absorption $\left(n_{2}=1.2 \cdot 10^{-9}\right.$ units CGSE, $n_{4}=-1.4 \cdot 10^{-12}$ units CGSE [3]) at $D=1 \mathrm{~mm}$, we have $P_{\mathrm{cr} 1} \approx 150 \mathrm{~W}, P_{\mathrm{cr} 2} \approx 1830 \mathrm{~W}$, $P_{\mathrm{cr} 3} \approx 180 \mathrm{~W}$, and $P_{\mathrm{cr} 4} \approx 2160 \mathrm{~W}$. The luminescence intensity of the streamer doing along the channel is $P$ $10 \mathrm{~kW}$ [5], therefore, at streamer discharge the condition of light self-channelling by the effect threshold $P_{\mathrm{s}}>P_{\mathrm{cr}}$ is met with significant reserve.

According to theoretical $[6,7]$ and experimental data [8], the process of radiation self-trapping results in plasma displacement of non-equilibrium carrier out of the light channel, i.e. the region of strong electric field, hence - in decrease of current value to lower than the destruction threshold. It is consistent with the absence of destructions in the channel of semiconductor streamer discharge [1] and could be one of the reasons for its nondestructive character.

Let us estimate the sizes of self-constricted beam in the medium under study, where the channel radius $R$ oscillates and depends on the beam size [2]. The analysis of dependence $R\left(P_{\mathrm{cr}}\right)$, taking into account the criterion (1) and experimental data for nonlinearity coefficient at light excitation [3], indicates the presence of two characteristic minima (branches) of this dependence
(Fig. 1), moreover, for $P_{\mathrm{cr} 1}$ criterion the branches are lower than for $P_{\mathrm{cr} 2}$.

The branch in the region $P_{\mathrm{cr}}=200 \ldots 300 \mathrm{~W}$ for the case $P_{\text {crl }}$ corresponds to the least influences by parameters of wave surface $(b)$. In this relation, it represents the greatest interest from the viewpoint of numerical modelling, which is subjected to the least influence of parameters inherent to the wave surface. Here, $b$ is the proportionality coefficient depending on $R_{f}^{2}=b R_{0}^{2} /|Q| \mid$, where $Q=n_{2} E^{2} / n_{0}-\left(k R_{0}\right)^{-2}, R_{f}-$ curvature radius of wave surface, $k$ - wavenumber, $R_{0}=D / 2-$ radius of initial beam. The increase in the factor of nonlinearity $n_{2}$ within the limits of one order (in comparison with the known data for crystals $\mathrm{CdS}$ ) results in reduction of the threshold power and the crosssection sizes of the light channel up to the values corresponding to parameters of streamer $P_{\mathrm{cr}} \leq$ $10 \ldots 30 \mathrm{~W}, R \leq 10 \mu \mathrm{m}$ (Fig. 2). Similar changes take place with decrease in $n_{4}$ coefficient from $\sim 10^{-12}$ to $10^{-14}$ units CGSE.

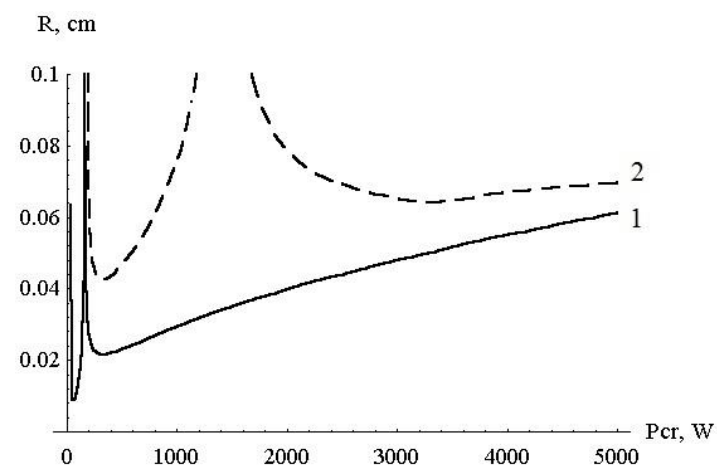

Fig. 1. Spatial-energetic characteristics of light selfchannelling effect for the criteria $P_{\mathrm{cr} 1}$ (1) and $P_{\mathrm{cr} 2}$ (2). $n_{2}=1.2 \cdot 10^{-9}$ units CGSE, $n_{4}=-1.4 \cdot 10^{-12}$ units CGSE, $b=1$.

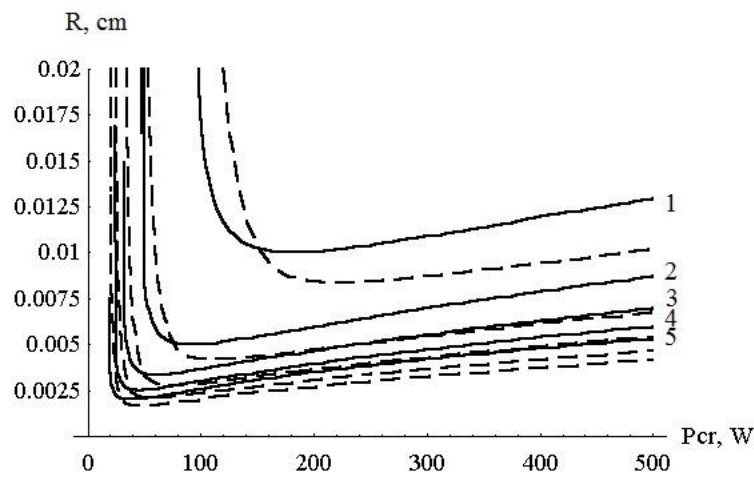

Fig. 2. Dependence of the light channel radius on threshold intensity at varying nonlinearity coefficients for spherical (solid lines) and cylindrical (dashed lines) beams: $n_{2}=2 \cdot 10^{-9}$ $(1), 4 \cdot 10^{-9}(2), 6 \cdot 10^{-9}(3), 8 \cdot 10^{-9}(4), 10^{-8}(5)$ units CGSE; $n_{4}=$ $=-10^{-12}$ units CGSE. 
Using the example of cadmium sulphide crystals, we calculated anisotropy of nonlinear susceptibility of the fifth order $\chi^{(5)}$, defining threshold and spatial properties of light self-channelling. Behavior of the system in strong electric field can be simulated by using incompletely symmetrical sixth-rank tensor of $\left[\left[\mathrm{V}^{2}\right]^{3}\right]$ type [9] or $\left[\mathrm{V}^{2}\right]\left[\left[\mathrm{V}^{2}\right]^{2}\right][10]$ - by analogy with the tensor of electro-optic effect (this tensor is known to be asymmetrical). Let us study the former case; the results of simulation, taking into account the second tensor, are similar. Spatial distribution $\chi^{(5)}$ corresponding to convolution of the given tensor in the plane of crystal related to the $(1 \overline{0} 10)$ type is determined by the expression

$\chi_{(1010)}^{(5)}=\chi_{111} \sin ^{6} \theta+\chi_{333} \cos ^{6} \theta+$

$+3\left(\chi_{113}+4 \chi_{155}\right) \sin ^{4} \theta \cos ^{2} \theta+$

$+3\left(\chi_{133}+4 \chi_{355}\right) \sin ^{2} \theta \cos ^{4} \theta$.

In approximation $\chi_{133} \approx \chi_{355} \approx \chi_{333}, \chi_{133} \approx \chi_{155} \sim$ $0.1 \chi_{333}$, the relation (3) gives three directions, symmetrical relative to $\langle\overline{1} 210\rangle(\theta=\pi / 2)$, which are different from the discharge directions on the average by $\pm(2 \ldots .5)^{\circ}$. In the basal plane (0001) angular distribution $\chi^{(5)}$ has the form

$$
\begin{aligned}
& \chi_{(0001)}^{(5)}=16\left(\chi_{111}-\chi_{222}\right) \cos ^{6} \varphi+ \\
& +24\left(\chi_{222}-\chi_{111}\right) \cos ^{4} \varphi+ \\
& +9\left(\chi_{111}-\chi_{222}\right) \cos ^{2} \varphi+\chi_{222}
\end{aligned}
$$

and gives the local maxima in the direction of $\langle 1 \overline{0} 10\rangle$ type in a rather wide range $+\infty>\chi_{111}>\chi_{222}$. The analysis of both relations (3) and (4) shows that spatial anisotropy of nonlinear susceptibility under consideration takes place, and hence, the self-channeling effect corresponds approximately to streamer orientation (with the accuracy pointed above). It indicates that the given effect conditions not only crystallographic orientation, but also thread-like shape of streamer discharge.

Similar calculations for cubic semiconductors (of $\overline{4} 3 \mathrm{~m}$ symmetry) using the forth-rank tensors, for example $\left[\mathrm{V}^{2}\right]^{2}$ or $\mathrm{V}\left[\mathrm{V}^{3}\right]$, result in localization of distribution maxima of nonlinear susceptibility in $\langle 110\rangle$ directions, corresponding to streamer orientation in $\mathrm{CdTe}, \mathrm{GaAs}$ and InP crystals with accuracy of several degrees.

It should be noted that in the experimentally obtained spatial picture (direction system) of streamer discharges in hexagonal semiconductors, in particular [1, $11,12]$, there are no basic symmetry elements of $6 \mathrm{~mm}$ type, except for prismatic planes, where they are localized. Asymmetry of the picture observed is apparent (within the range $0,5 \ldots 1^{\circ}$ ) relatively to the axis of the sixth order. It indicates the changes (distortions) in crystal symmetry as well as the fact that in the chosen conditions there appear two families of discharges simultaneously - positively and negatively directed ones (of different poles) according to the statements [13]. In cubic semiconductors, there are also distortions of lattice symmetry appearing in incomplete coincidence of discharge path with the directions [110]. In this relation, spatial orientation of streamers reflects the point group of not only initial, i.e. undisturbed crystal (familiar Neyman's principle and German's theorem), but also the result of field interaction with the medium and corresponds to the general principle of the Curie symmetry superposition - dissymmetry addition principle. Therefore, application of "non-destructive" streamer discharges is a sophisticated and in some cases unique tool for investigation of electric, nonlinear-optic, crystallographic and a number of other properties of solids in strong fields.

Thus, the effect of light self-influence in selfchannelling condition (wave-guide mode) at streamer discharge in media with cubic and fifth-order nonlinearities has been considered. In the course of selfconsistent problem, the threshold and spatial properties of light self-channelling for hexagonal and cubic semiconductors have been estimated. These data are close to corresponding characteristics of streamer tracks. Nonlinear interaction of discharge radiation with semiconductor in the waveguide mode results in threadlike shape of streamer and decreases (removes) fractures of crystal lattice. Symmetry for crystallographic directions system of streamer discharges in wide-gap semiconductors corresponds to the general principle of symmetry superposition.

\section{References}

1. N.G. Basov, A.G. Molchanov, A.S. Nasibov, A.Z. Obidin, A.N. Pechenov, and Yu.M. Popov, Solid body streamer lasers // Zhurnal eksperiment. teor. fiziki, 70(5), p. 1751-1761 (1976), in Russian.

2. S.A. Akhmanov, A.P. Sukhorukov, and R.V. Khohlov, On self-focusing and selfchanneling of intensive light beams in nonlinear medium // Zhurnal eksperiment. teor. fiziki, 50(6), p. 1537-1549 (1966), in Russian.

3. A.A. Borshch, M.S. Brodin, F.N. Marchevskiy, and V.N. Semioshko, Nonlinear susceptibility anisotropy of cadmium sulphide crystals // Quantovaya elektronika, 11(10), p. 2041-2048 (1984), in Russian.

4. A.A. Borshch, V.I. Volkov, and A.I. Mitskan, Two-wave interaction of nanosecond laser pulses in CdTe crystals and origin of their nonlinearity // Quantovaya elektronika, 22(4), p. 383-385 (1995), in Russian.

5. A.S. Nasibov, A.Z. Obidin, A.N. Pechenov, Yu.M. Popov, and V.A. Frolov, Generation of 
optical radiation in the direction of streamer spreading // Kratkiye soobshcheniya po fizike, 11, p. 39-42 (1978), in Russian.

6. Ya.L. Bogomolov, S.F. Lirin, V.E. Semenov, and A.M. Sergeev, Ionization self-trapping of superstrong electromagnetic waves in plasma // Pisma $v$ zhurnal eksperiment. teor. fiziki, 45(11), p. 532-535 (1987), in Russian.

7. L.V. Keldysh, Ionization in the field of strong electromagnetic wave // Zhurnal eksperiment. teor. fiziki, 47, p. 1945-1957 (1964), in Russian.

8. G.M. Zverev, E.K. Maldutis, and V.A. Pashkov, On self-focusing of laser light in solid dielectrics // Pisma $v$ zhurnal eksperiment. teor. fiziki, 9(1), p. 108-110 (1969), in Russian.
9. F.G. Fumi, The elastic coefficients in trigonal and hexagonal crystals // Phys. Rev. 86(4), p. 561 (1952).

10. K. Vedam, and R. Srinivasan, Non-linear piezooptics // Acta Crystallogr. 22(5), p. 630-634 (1967).

11. V.P. Gribkovskii, Streamer light in semiconductors // Zhurnal prikladnoy spektroskopii, 40(5), p. 709718 (1984), in Russian.

12. V.P. Gribkovskiy, V.V. Parashchuk, and K.I. Rusakov, On crystallographic orientation of streamer discharges // Zhurnal tekhnich. fiziki, 64 (11), p. 169-171 (1994), in Russian.

13. M.I. Diakonov, and A.S. Furman, Discharge relaxation in anisotropic medium and in media with low dimension // Zhurnal eksperiment. teor. fiziki, 92(3), p. 1012-1020 (1987), in Russian. 